\title{
Non-invasive measurements to stratify cardiovascular disease risk in psoriasis patients
}

Philippa Dickison, Jonathan J Peek, Grace Swain, Saxon D Smith

\section{Background and objectives \\ Psoriasis is associated with cardiovascular disease (CVD) risk factors and mortality. The aims of this study were to identify CVD risk in patients with psoriasis, and to determine their awareness of the comorbidities.}

\section{Methods}

Patients with psoriasis and controls had their inter-arm blood pressure, weight, height and waist circumference measured at their routine clinic appointment. Those with psoriasis also completed a survey regarding awareness of the comorbidities.

\section{Results}

A total of 179 patients with psoriasis and 115 control patients participated in the study. Patients with psoriasis were significantly more likely to be obese (odds ration [OR] 6.3). An inter-arm blood pressure difference $>10 \mathrm{mmHg}$ was more likely in patients with psoriasis for systolic (OR 1.9) and diastolic (OR 2.3) readings. Survey data showed that 47 (26.3\%) psoriasis patients knew that psoriasis was associated with being overweight.

\section{Discussion}

On the basis of anthropologic measurements, patients with psoriasis have a higher risk of CVD, compared with non-psoriasis patients. There is inadequate knowledge among psoriasis patients regarding the comorbidities of psoriasis.
PSORIASIS IS AN immune-mediated systemic disorder affecting $1-5 \%$ of the population. ${ }^{1}$ Psoriasis typically manifests with chronic erythematous plaques, but is also associated with many conditions, including psoriatic arthritis, metabolic syndrome and Crohn's disease. ${ }^{2}$ Additionally, moderate-to-severe psoriasis increases cardiovascular disease (CVD) mortality, and is associated with an increased prevalence of dyslipidaemia, hypertension and diabetes..$^{2-4}$ Elevated body mass index (BMI) and smoking have also been found to increase the onset of psoriasis..$^{5,6}$ This association is most likely because psoriasis results in systemic inflammatory changes. ${ }^{2}$

Recent data have suggested that patients with psoriasis are not aware of these comorbidities. ${ }^{78}$ In a survey of patients with psoriasis, Skiveren et $\mathrm{al}^{7}$ found that only 11.9-15.3\% correctly answered questions regarding the association with CVD. Younger ages, being treated with a biological agent or being part of a patients' association corresponded with an increased awareness. ${ }^{78}$ Limited knowledge of the comorbidities may confer an increased risk of the associated comorbidities, as management of psoriasis requires patients to alter their behaviours and lifestyle.

Lee et $\mathrm{al}^{9}$ showed that children with psoriasis have a significantly increased risk of central adiposity, compared with controls. The study recommended that waist-to-height ratio (WtHR) could be routinely used to detect and track CVD risk in children with psoriasis. Similarly, differences in blood pressure between arms, typically a result of atherosclerosis, is known to correspond to the presence or increased risk of CVD, chronic renal disease, hypertension and peripheral vascular disease.$^{10}$ Clark et al ${ }^{11}$ found that measuring blood pressure in both arms was a reliable measure for predicting CVD risk in the primary care setting. The study showed that an inter-arm difference in blood pressure of $\geq 10 \mathrm{mmHg}$ correlated with an increased risk of CVD events over 10 years. Inter-arm blood pressure measurements are therefore a valuable indicator for CVD risk. This non-invasive method can be easily applied in the clinical setting.

Methods for stratifying CVD risk in adult patients with psoriasis have not been previously established. The aims of this study were to evaluate the CVD risk among adult psoriasis patients in an outpatient setting, assess their awareness of the associated comorbidities, and determine suitable measures that can be easily used in the clinical setting to identify CVD risk.

\section{Method}

\section{Study design and participants}

This study was approved by the Northern Sydney Local Health District Human Research and Ethics Committee (HREC; reference LNR/15/HAWKE/197). The case-control study was carried out at a dermatology clinic in a tertiary referral hospital in Sydney, a phototherapy unit in the same hospital and a private dermatology clinic in an outer metropolitan area in New South Wales, Australia, between February 2016 and July 2017. This study considered two patient groups: those with clinically diagnosed psoriasis, and controls. Sequential patients were approached at the clinics and informed of the study. All English- 
speaking patients aged $\geq 18$ years who had no contraindications for measuring blood pressure in both arms (amputation, lymphoedema, dialysis fistula) were eligible to participate. For control patients, having any other inflammatory disease was an indication for exclusion. The survey contained questions encompassing cardiovascular parameters, and patients' current understanding of disease aetiology and awareness of associated comorbidities. Sample size was calculated a priori assuming that $33 \%$ of controls were on antihypertensive agents or had hypertension (blood pressure $\geq 140 / 90$ $\mathrm{mmHg}){ }^{12}$ A total of 274 participants was required to test an odds ratio (OR) of 2 .

\section{Data collection}

\section{Cardiovascular parameters}

Consenting participants completed demographic and medical information in addition to physical measurements (height, weight, waist circumference and blood pressure from both arms). Prior to their consultation, blood pressure was measured from each arm, one minute apart, while the patient was in a comfortable sitting position. The appropriate cuff size was based on the accepted range indicated on the cuff. The cuff was placed directly on the upper arm. Manual sphygmomanometers in the consultation rooms (with Littman stethoscopes [3M, Minnesota, US]) were used and the same machine was used on both arms.

\section{Psoriasis parameters}

Additional information was collected from participants with psoriasis regarding current treatment and psoriasis severity. All treatments at the time of recruitment were recorded. Biological agents included medications directed against cytokines, such as tumour necrosis factor (TNF)alpha (infliximab, adalimumab) or interleukins 12, 23 or 17 (ustekinumab, ixekizumab) available through the Pharmaceutical Benefits Scheme (PBS). ${ }^{13}$ Severity was assessed through standardised measures: Psoriasis Area and Severity Index (PASI), a six-point Static Physicians Global Assessment (sPGA) and body surface area (BSA) percentage. BSA was divided into $<5,5-10,11-20$ and $>20 \%$. Severe psoriasis was identified in patients with a PASI $>10$.

\section{Awareness}

Bardazzi et al ${ }^{8}$ created a survey that assessed patients' awareness and understanding of psoriasis. This survey was found to be valid in assessing patients' awareness of clinical course, diagnosis and pathogenesis of psoriasis, and sources of information about psoriasis. In addition to shortening the survey developed by Bardazzi et al, we adapted it in response to feedback from the HREC. Questions were presented to participants in the form of a five-point Likert-style rating to assess psoriasisrelated knowledge (Table 1). Sources of patient information were considered through a series of binary questions.

\section{Statistical analysis}

Data were analysed with IBM SPSS

Statistics Version 22. Descriptive data were presented as measures of central tendency and variability. Continuous variables were

\section{Table 1. Likert-style questions asked to test awareness}

All questions had five options, displayed beneath question 1

1. Is psoriasis infectious?

\begin{tabular}{l}
\multicolumn{1}{c}{ Yes I think so I'm not sure Probably not } \\
2. Is psoriasis inherited through genes? \\
\hline 3. Can psoriasis affect other organs in the body? \\
4. Do you think psoriasis increases the chance of heart disease? \\
\hline 5. Is psoriasis associated with allergies? \\
\hline 6. Can sun exposure improve psoriasis? \\
7. Can infections worsen psoriasis? \\
8. Can smoking worsen psoriasis? \\
9. Can alcohol worsen psoriasis? \\
10. Can stress and anxiety cause or worsen psoriasis?
\end{tabular}

11. Are there other diseases that can be associated with psoriasis?

12. Do you think psoriasis can increase the chances of being overweight?

13. Does psoriasis increase the chances of anxiety or depression?

14. Have you been informed on possible drugs or care options for psoriasis?

15. Do you know what metabolic syndrome means?

\section{What sources of information have you heard about psoriasis from?}

\begin{tabular}{lccc}
\hline Newspapers & Yes & No \\
\hline TV & Yes & No \\
\hline Radio & Yes & No \\
\hline Internet & Yes & No \\
\hline Friends/relatives & Yes & No \\
\hline Family doctor & Yes & No \\
\hline Pharmacist & Yes & No \\
\hline
\end{tabular}


analysed against categorical variables with independent sample t-test. Categorical variables were analysed using a chi-square test and Fisher's exact test. ORs were calculated using binary regression with 95\% confidence interval (CI) to analyse associations between categorical variables of interest. A $P$ value of $<0.05$ was considered significant.

\section{Results}

\section{Demographic data}

A total of 179 patients with psoriasis and 115 control patients participated in the study. The demographic characteristics between the groups were not significantly different, apart from age; the mean age of patients with psoriasis was 52.3 years (standard deviation $[\mathrm{SD}]=15.1$ ), whereas the mean age of the control patients was 57.2 years $(\mathrm{SD}=19.7 ; P=0.017)$ (Table 2$)$. Additionally, the ethnicities of 13 patients in the control group did not fall into Caucasian, South Asian, East Asian or Middle Eastern ('All other' in Table 2; $P=0.003)$.

\section{Psoriasis versus controls}

When compared with control patients, those with psoriasis were significantly more likely to have an increased BMI $(P<0.001)$, a WtHR $>0.5(P<0.001)$ and blood pressure $>140 / 90 \mathrm{mmHg}$ at the time of testing $(P=0.002$; Table 2$)$. Furthermore, an inter-arm blood pressure difference $>10$ was significantly more prevalent in the patients with psoriasis for systolic $(P=0.04)$ and diastolic $(P=0.04)$ readings.

\section{Psoriasis data}

Sixty-three (35.2\%) patients with psoriasis had a PASI $>10$. Almost one-quarter of the psoriasis patients in this study were on biological agents $(n=42,23.5 \%)$ (Table 3 ). Patients on biological agents were less likely to have a PASI >10 (OR: 0.2; 95\% CI: $0.1-0.6 ; P=0.003)$; patients on topical agents were 3.2 times more likely to have a PASI >10 (95\% CI: $1.4-7.2 ; P=0.004)$. Not being on any treatments, including biological agents, was significantly associated with increased BMI, blood pressure $>140 / 90$ or WtHR $>0.5$.

\section{Sources of information}

Patients' sources of information about psoriasis are shown in Table 3.

\section{Awareness data}

Regarding general psoriasis knowledge, 171 (95.5\%) patients correctly identified that psoriasis was not infectious, 152

$(84.9 \%)$ identified that it was an inherited condition and 167 (93.3\%) identified that psoriasis improves with sun exposure (Figure 1A).

Ninety-eight (54.7\%) patients thought that psoriasis affected other organs, apart from skin. The number of patients who correctly identified that psoriasis was a CVD risk factor was 91 (50.8\%), and 47 (26.3\%) for being overweight(Figure 1B).

For the survey questions regarding mental health, 170 (95\%) patients identified that stress and anxiety worsen psoriasis, and 152 (84.9\%) identified that psoriasis increases the chance of depression and anxiety (Figure 1C).

Patients with psoriasis who had a WtHR $>0.5$ were more likely to identify that psoriasis was a risk factor for heart disease $(P=0.02)$. There were no other psoriasis factors or CVD risk factors significantly associated with the survey questions.

\section{Discussion}

This study corroborates previous findings by clearly demonstrating that patients with psoriasis are significantly more likely to be overweight or obese, and have hypertension or an increased WtHR when compared with controls. ${ }^{2,4,5,9,12}$ Although the psoriasis group was significantly younger than the control group, this study found a relationship between psoriasis, hypertension, obesity and increased WtHR.

All of the patients in our study who were on biological agents accessed them through the PBS, meaning they previously had moderate-to-severe psoriasis (PASI >10). ${ }^{13}$ This study demonstrated that $\mathrm{BMI}$ and other CVD risk factors were not significantly increased in patients who were on biological agents. This suggests that an improvement in PASI may also correspond with an improvement in BMI and, therefore, other CVD risk factors.
This finding complements recent research showing that improvement in psoriasis through the use of anti-TNF- $\alpha$ biological agents is associated with improved endothelial function. ${ }^{14} \mathrm{~A}$ large controlled study is required to further evaluate this relationship.

Compared with the study by Skiveren et al, ${ }^{7}$ our study found a higher awareness of CVD. However, the questions regarding smoking, heart disease and, especially, being overweight demonstrate a lack of knowledge and, most likely, education. This is concerning considering the prevalence of CVD risk factors in the psoriasis population. An increased level of awareness is important in psoriasis, as management involves not only the skin but lifestyle factors to reduce the CVD risk. Almost all the patients with psoriasis surveyed identified the link between psoriasis and stress, anxiety and the increased likelihood of mood disorders. These results are most likely to be a reflection of the loss of confidence and experience of stigma that has been identified previously by psoriasis patients. ${ }^{15}$

General practitioners (GPs) and dermatologists were most frequently identified as sources of information regarding psoriasis. This indicates that there is a role for medical professionals to detect, educate and holistically manage both psoriasis and the CVD comorbidities. The Australian consensus of treatment goals for psoriasis advocate for the involvement of GPs in the management of psoriasis and the associated comorbidities. ${ }^{16}$ There are, however, no specific guidelines regarding non-invasive risk stratification or management of CVD risk factors in patients with psoriasis in the Australian, European or US guidelines. ${ }^{17,18}$

Rather than complicate the recommendations available through the addition of invasive investigations, our study has clearly demonstrated the utility of simple, non-invasive anthropometric investigations. WtHR $>0.5$ has been shown to be a useful tool for measuring body fat distribution and future CVD. ${ }^{19,20}$ Similarly, an inter-arm difference of $10 \mathrm{mmHg}$ in blood pressure was found to correspond with cardiovascular events. ${ }^{10}$ The WtHR and inter-arm blood pressure are valuable 


\section{Table 2. Demographic characteristics and comparison of cardiovascular risk factors between the psoriasis and} control groups

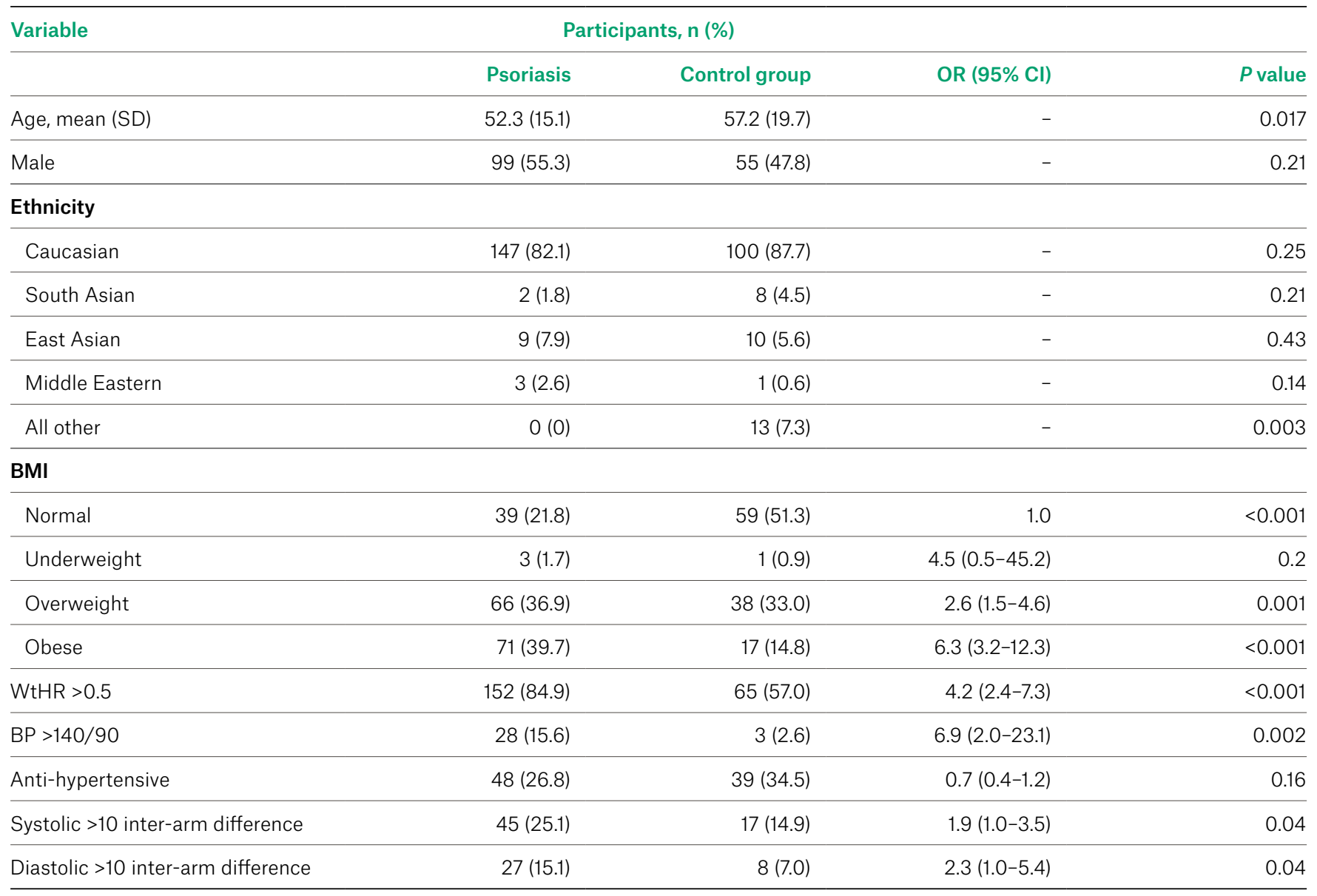

$B M I$, body mass index; $B P$, blood pressure; $O R$, odds ratio; SD, standard deviation; WtHR, waist-to-height ratio

predictors of CVD and are therefore very useful for patients with psoriasis. The measurements can easily be carried out in any practice.

The limitations of this study are associated with the collection sites. First, the different sphygmomanometers and the 'white coat' effect may have affected the blood pressure measurements. However, the inter-arm data was not affected as the same machine was used for each arm. Second, the patients with psoriasis were recruited from specialist rooms and outpatient clinics. These patients were more likely to have severe disease or require systemic treatments than those managed by non-dermatologists. Third, there were fewer control patients than patients with psoriasis. Despite this, the groups were well matched. That this study has clearly demonstrated increased CVD risk factors among patients with psoriasis underscores the need for further research regarding CVD detection and risk reduction in these patients.

Our study demonstrated the degree to which psoriasis patients at the study sites already have increased CVD risk factors. This was shown through interarm blood pressure differences and WtHR. Inter-arm blood pressure, waist and height measurement are easily performed in dermatology clinics and general practices, and can indicate to both patient and clinician when further management of CVD risk factors is required. Psoriasis patients in this study were also shown to be frequently unaware of the association between psoriasis and CVD risk factors, which may deleteriously affect any necessary lifestyle modifications. Clinicians should be encouraged to perform these noninvasive measurements, manage increased CVD risk factors identified and increase the awareness in their patients of the association between psoriasis and CVD.

\section{Implications for general practice}

- GPs need to remain vigilant regarding CVD risk factors in patients with psoriasis, as they are at increased risk as demonstrated in this study and previous literature.

- Inter-arm blood pressure and WtHR are useful in measuring risk factors in 


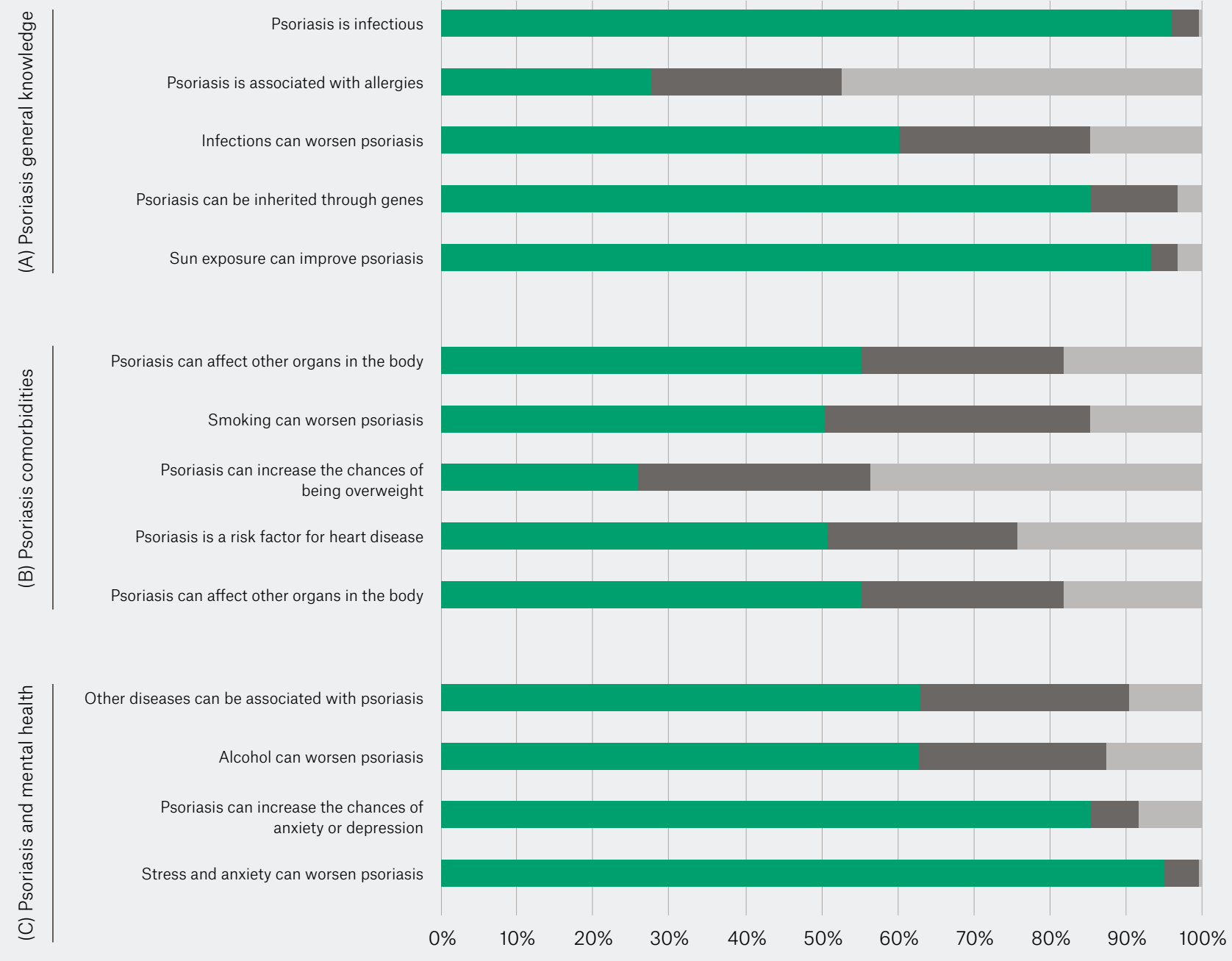

Proportion of answers

Correct Unsure Incorrect

Figure 1. Cumulative bar chart of responses to survey questions

patients with psoriasis and are easily performed in general practice.

- Despite the well-established link between psoriasis and CVD risk factors, patients with psoriasis are not necessarily aware of this association, which could have implications on their lifestyle and risk reduction management. GPs play an important role in the education of these patients.

\section{Authors}

Philippa Dickison MD, PhD candidate, Sydney Medical School, University of Sydney, Sydney, NSW: Department of Dermatology, Royal North Shore Hospital, St Leonards, NSW. philippa.dickison@ sydney.edu.au

Jonathan J Peek, BA, medical student, Sydney Medical School, University of Sydney, Sydney, NSW

Grace Swain, BSc (Hons), medical student, Sydney Medical School, University of Sydney, Sydney, NSW

Saxon D Smith MBChB, Clinical Associate Professor, Sydney Medical School, University of Sydney, Sydney,
NSW; Department of Dermatology, Royal North Shore Hospital, St Leonards, NSW; Skin Cancer and Dermatology Centre, Gosford, NSW

Competing interests: None.

Provenance and peer review: Not commissioned, externally peer reviewed.

\section{Acknowledgements}

The authors would like to acknowledge Dr Luke Dan for his role in the creation and piloting of the initial survey distributed to psoriasis patients. 


\section{Table 3. Treatment, severity and sources of information of the psoriasis patients}

n (\%)

\begin{tabular}{ll}
\hline & $\mathrm{n}(\%)$ \\
\hline Topical therapy & $123(68.7)$ \\
\hline Phototherapy & $84(46.9)$ \\
\hline Oral & $46(25.7)$ \\
\hline Biologic & $42(23.5)$ \\
\hline PASI $>10$ & $63(35.2)$ \\
\hline BSA & \\
\hline Mild & $64(35.8)$ \\
\hline Moderate & $29(16.2)$ \\
\hline Severe & $28(15.6)$ \\
\hline Extensive & $56(31.3)$ \\
\hline
\end{tabular}

\section{Sources of information}

\begin{tabular}{lc} 
Newspapers & $38(21.2)$ \\
\hline TV & $57(31.8)$ \\
\hline Radio & $24(13.4)$ \\
\hline Internet & $120(67.0)$ \\
\hline Friends/relatives & $103(57.5)$ \\
\hline
\end{tabular}

Dermatologist or GP $153(85.5)$

Pharmacist

85 (47.5)

BSA, body surface area; GP, general practitioner; PASI, Psoriasis Area and Severity Index

\section{References}

1. World Health Organization. Global report on psoriasis. Geneva: WHO, 2016. Available at http://apps.who.int/iris/ bitstream/10665/204417/1/9789241565189_eng. pdf [Accessed 13 February 2018].

2. Boehncke WH, Schön MP. Psoriasis. Lancet 2015;386(9997):983-94. doi: 10.1016/S01406736(14)61909-7.

3. Samarasekera EJ, Neilson JM, Warren RB, Parnham J, Smith CH. Incidence of cardiovascular disease in individuals with psoriasis: A systematic review and meta-analysis. J Invest Dermatol 2013;133(10):2340-46. doi: 10.1038/jid.2013.149.

4. Armstrong AW, Harskamp CT, Armstrong EJ. The association between psoriasis and hypertension: A systematic review and meta-analysis of observational studies. J Hypertens 2013;31(3):43342. doi: 10.1097/HJH.0b013e32835bcce1.

5. Setty AR, Curhan G, Choi HK. Obesity, waist circumference, weight change, and the risk of psoriasis in women: Nurses' Health Study II. Arch Intern Med 2007:167(15):1670-75.
6. Armstrong AW, Harskamp CT, Dhillon JS, Armstrong EJ. Psoriasis and smoking: $A$ systematic review and meta-analysis. $\mathrm{Br} J$ Dermatol 2014;170(2):304-14. doi: 10.1111/ bjd.12670.

7. Skiveren J, Philipsen P, Therming G. Patients with psoriasis have insufficient knowledge of their risk of atherothrombotic disease and metabolic syndrome. Clin Exp Dermatol 2015:40(6):600-04. doi: 10.1111/ced.12628.

8. Bardazzi F, Amerio P, Amoruso G, et al. Investigating psoriasis awareness among patients in Italy: Validation of a questionnaire. Eur Rev Med Pharmacol Sci 2014;18(22):3435-52.

9. Lee A, Smith SD, Hong E, Garnett S, Fischer G. Association between pediatric psoriasis and waist-to-height ratio in the absence of obesity: A multicenter Australian study. JAMA Dermatol 2016;152(12):1314-19. doi: 10.1001/ jamadermatol.2016.3432.

10. Clark CE, Taylor RS, Shore AC, Ukoumunne OC, Campbell JL. Association of a difference in systolic blood pressure between arms with vascular disease and mortality: A systematic review and meta-analysis. Lancet 2012;379(9819):905-14. doi: 10.1016/S0140-6736(11)61710-8.

11. Clark CE, Taylor RS, Shore AC, Campbell JL. The difference in blood pressure readings between arms and survival: Primary care cohort study. BMJ 2012;344:e1327. doi: 10.1136/bmj.e1327.

12. Armstrong AW, Harskamp CT, Armstrong EJ. The association between psoriasis and obesity: A systematic review and meta-analysis of observational studies. Nutr Diabetes 2012;2:e54. doi: 10.1038/nutd.2012.26.

13. Department of Human Services. Psoriasis Severe chronic plaque psoriasis - Adult patients. Canberra: DoHS, 2018. Available at www. humanservices.gov.au/health-professionals/ enablers/severe-chronic-plaque-psoriasis [Accessed 13 February 2018].

14. Pina T, Genre F, Lopez-Mejias R, et al. Anti-TNFalpha therapy reduces retinol-binding protein 4 serum levels in non-diabetic patients with psoriasis: A 6-month prospective study. J Eur Acad Dermatol Venereol 2016;30(1):92-95. doi: 10.1111/ jdv.13005.

15. Baker CS, Foley PA, Braue A. Psoriasis uncovered - Measuring burden of disease impact in a survey of Australians with psoriasis. Australas J Dermatol 2013;54 Suppl 1:1-6. doi: 10.1111/ajd.12010.

16. Baker C, MacK A, Cooper A, et al. Treatment goals for moderate to severe psoriasis: An Australian consensus. Australas J Dermatol 2013;54(2):14854. doi: 10.1111/ajd.12014.

17. Kimball AB, Gladman D, Gelfand JM, et al. National Psoriasis Foundation clinical consensus on psoriasis comorbidities and recommendations for screening. J Am Acad Dermatol 2008;58(6):1031-42. doi: 10.1016/j. jaad.2008.01.006.

18. Richard MA, Barnetche T, Horreau C, et al. Psoriasis, cardiovascular events, cancer risk and alcohol use: Evidence-based recommendations based on systematic review and expert opinion. J Eur Acad Dermatol Venereol 2013;27 Suppl 3:2-11. doi: 10.1111/jdv.12162.

19. Ashwell M, Cole TJ, Dixon AK. Ratio of waist circumference to height is strong predictor of intra-abdominal fat. BMJ 1996;313(7056):559-60.
20. Ashwell M, Gibson S. Waist-to-height ratio as an indicator of 'early health risk': Simpler and more predictive than using a 'matrix' based on BMI and waist circumference. BMJ Open 2016;6(3):e010159. doi: 10.1136/bmjopen-2015010159. 